\title{
Spontaneous Tension Hemothorax in a Young Male with a Nuss Implant
}

\author{
Guorun Holm Jacobsen, Bodil Brandt and Sabrina Gade Ellesøe*
}

Department of Cardio-thoracic Surgery, Rigshospitalet, Blegdamsvej 9, 2100 København O, Denmark

\begin{abstract}
A 19-year-old male presented with upper abdominal pain, shortness of breath and loss of consciousness. X-rays revealed a tension hemothorax and a chest tube was inserted evacuating $2500 \mathrm{ml}$ of blood. Bleeding in the tube ceased after the initial evacuation and the patient was monitored closely and could be discharged in good health on the second day after admission. We hypothesize, that the tension hemothorax was preceded by a spontaneous pneumothorax, which caused a tear in scar tissue following the Nuss procedure five months previously.
\end{abstract}

Keywords: Nuss procedure; Spontaneous tension hemothorax; Emergency thoracoscopy

\section{Introduction}

Tension hemothorax is a rare condition characterized by mediastinal displacement caused by a large volume of blood within the pleural cavity. Spontaneous cases are extremely rare and there has only been, to our best knowledge, two cases reported in the English literature $[1,2]$. In contrast, primary spontaneous pneumothorax is common with a reported male incidence of $18-28$ pr. 100.000 per year [2]. In $0.5-12 \%$ of these cases, spontaneous pneumothorax can be complicated by a hemothorax [3]. Spontaneous hemopneumothorax is a rare condition characterized by accumulation of blood within the pleural cavity in association with a spontaneous pneumothorax [4]. Although extremely rare, spontaneous hemopneumothorax can become life threatening due to the hemodynamic instability and ventilatory collapse [5].

This case presents a young male developing a spontaneous tension hemothorax, preceded by a spontaneous pneumothorax which we hypothesize caused a tear in scare tissue following the NUSS procedure five months prior. This is, to our best knowledge, the first incidence of development of a tension hemothorax in a Nuss patient.

\section{Case Presentation}

A 19-year-old male was admitted to the emergency department in a secondary centre. Ten hours prior he experienced reduced endurance and dyspnoea during a running session. Over the following seven to eight hours the symptoms worsened and he gradually developed right-sided upper abdominal pain radiating to the back, shortness of breath and loss of consciousness. There was no history of trauma before onset of symptoms. Five months earlier he had undergone the NUSS procedure using a one 11-inch. Pectus bar and the procedure as well as the postoperative stay had been unremarkable.

At admission, physical examination revealed pallor, hypotension (96/58 $\mathrm{mmHg}$ ), tachycardia (109 beats/minute), tachypnea (1735 beats/minute) and frequent desaturation. ECG was normal. No traumatic injuries or abnormalities were noted upon inspection of chest, back or abdomen.

Tension pneumothorax was suspected why a peripheral venous catheter was inserted at intercostal space two revealing that blood, not air, was under pressure. The procedure relieved the respiratory distress instantly. The X-ray showed right-sided hemothorax and mediastinal displacement to the left, but normal position of the Nuss implant (Figure 1A). A chest tube was inserted and $2500 \mathrm{ml}$ was evacuated from the right pleural cavity.

Biochemical testing showed a drop of haemoglobin to $5.6 \mathrm{mmol} / \mathrm{L}$. A CT angiography was performed, but did not reveal any site of active bleeding. The patient was transferred to our tertiary centre, where X-ray upon arrival (12 hours after insertion of the chest tube) showed minimal fluid accumulation in the right pleural cavity and full expansion of the lungs (Figure 1B). TTE did not reveal any cardiac cause for the hemothorax. Therefore he was monitored closely for drop in haemoglobin level and hemodynamic instability but his vital signs remained stable and the chest tube could be removed two days after admission. The follow up X-ray was normal besides a small apical pneumothorax and the patient was discharged two days after admission.

\section{Discussion}

Life threatening tension hemothorax is most often caused by penetrating or blunt trauma to the chest or as a complication to a procedure [4]. Spontaneous tension hemothorax is extremely rare and has, to our best knowledge, only been reported twice in the English literature $[1,2]$. Here, we report the first incidence of a patient with tension hemothorax, who had undergone the Nuss procedure five months prior.

Spontaneous pneumothorax is by far the most common cause of hemothorax, where $0.5 \%-12 \%$ of all cases are complicated by a hemothorax $[3,4]$. Spontaneous hemothorax is defined as accumulation of more than $400 \mathrm{ml}$ of blood in the pleural cavity in association with a spontaneous pneumothorax [4]. The bleeding can be caused by a torn adhesion between the parietal and visceral pleura, rupture of vascularized bullae and underlying lung parenchyma or an aberrant vessel between the parietal pleura and bullae. More rare causes of hemothorax are coagulopathy, aortic dissection or rupture, neoplasia or endometriosis [4]. Kelly et al. [6] evaluated 2,378 pectus excavatum patients, where 1,215 underwent the pectus excavatum repair [6]. They reported four late hemothoraces. However, two were secondary to trauma, whereas the source of bleeding was not identified in the remaining two cases $(0.16 \%)$ and therefore could be considered as a spontaneous hemothorax.

Upon the patient's arrival, we suspected bar displacement as a cause

*Corresponding author: Sabrina Gade Ellesøe, Department of Cardio-thoracic Surgery, Rigshospitalet, Blegdamsvej 9, 2100 København O, Denmark, Tel: 004529925178; E-mail: sabrina.gade.ellesoee@regionh.dk

Received January 10, 2017; Accepted February 16, 2017; Published February 20, 2017

Citation: Jacobsen GH, Brandt B, Ellesøe SG (2017) Spontaneous Tension Hemothorax in a Young Male with a Nuss Implant. J Pulm Respir Med 6: 394. doi: 10.4172/2161-105X.1000394

Copyright: ( 2017 Jacobsen $\mathrm{GH}$, et al. This is an open-access article distributed under the terms of the Creative Commons Attribution License, which permits unrestricted use, distribution, and reproduction in any medium, provided the original author and source are credited. 


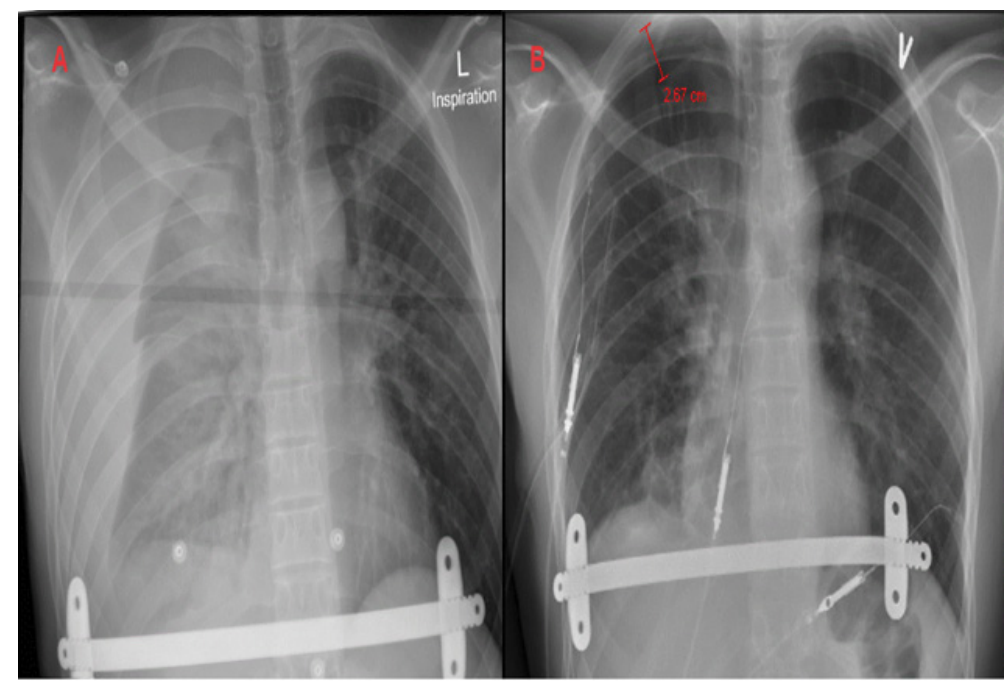

Figure 1: A: X-ray at secondary centre with right-sided tension hemothorax and mediastinal shift. B: X-ray at our centre 24 hours after debut of symptoms showing the chest tube, pleural cavity and a small apical pneumothorax.

of the hemothorax. Bar displacement or friction between bar and ribs has been shown to cause hemothorax as reported in earlier cases $[7,8]$. However, X-ray performed at admission showed normal position of the chest implant and the CT angiography could not identify any source of active bleeding.

Therefore, we hypothesize, that the tension hemothorax was caused by a spontaneous pneumothorax causing a tear in scar tissue following the Nuss procedure five months prior. At our tertiary centre, a patient with relevant trauma to the thorax and evacuation of $1000 \mathrm{ml}$ of blood or more upon chest tube insertion will indicate emergency thoracoscopy or thoracotomy depending on the clinical appearance of the patient. However, this patient was received in a secondary trauma unit and already had a chest tube inserted prior to transfer to our center. He did not have a history of trauma and there was no additional bleeding in the chest tube after admission to our center. Due to the uneventful condition of the patient after primary evacuation of blood as well as well drained pleural cavities 12 hours after chest tube insertion, we chose to monitor the patient closely instead of doing an emergency thoracotomy. He could be discharged in good health two days after admission.

Although extremely rare, a spontaneous pneumothorax can develop into a life threatening tension hemothorax that requires immediate intervention as well as close monitoring. Therefore we recommend that cases with spontaneous tension hemothorax should always be transferred to a thoracic surgery unit where experienced surgeons can be evaluate the patient and perform an emergency thoracoscopy if necessary. While only a very small fraction of the general population has a Nuss implant, the majority of this particular population consists of very young primarily lean males, who have a higher risk of spontaneous pneumothorax. These young males are at higher risk of developing a hemopneumothorax because of the potential formation of pleural scar tissue.

Fallon et al. [9] found that use of a stabilizer decreased the risk of bar migration, readmissions and reoperations and we have utilized bar stabilizers since 2000. However, Fallon and colleagues did not report any case of spontaneous tension hemothorax late after bar implant and therefore it is uncertain whether placement of a stabilizer decreases the risk of this extremely rare complication. Although very rare it is important, to bear this complication in mind when receiving patients with sudden onset of chest pain or dyspnea especially younger patients who have undergone thoracic surgery.

\section{References}

1. Patterson BO, Itam S, Probst F (2008) Spontaneous tension haemopneumothorax. Scand J Trauma Resusc Emerg Med 3: 3-5.

2. Johnson M, French S, Cornwall D (2014) An unusual case of primary spontaneous tension pneumothorax in a Jamaican female. West Indian Med J 63: $274-277$

3. Kakaris S, Athanassiadi K, Vassilikos K, Skottis I. (2004) Spontaneous hemopneumothorax : a rare but life-threatening entity. Eur J Cardio-Thoracic Surg 25: 856-858.

4. Ali HA, Lippmann M, Mundathaje U, Khaleeq G (2008) Spontaneous hemothorax: A comprehensive review. Chest 134: 1056-1065.

5. Tay CK, Yee YC, Asmat A (2015) Spontaneous hemopneumothorax: Our experience with surgical management. Asian Cardiovasc Thorac Ann 23: 308-310.

6. Kelly RE, Goretsky MJ, Obermeyer R, Kuhn MA, Redlinger R, et al. (2010) Twenty-one years of experience with minimally invasive repair of pectus excavatum by the Nuss procedure in 1215 patients. Ann Surg 252: 1072-1081.

7. Adam LA, Lawrence JL, Meehan JJ (2008) Erosion of the Nuss bar into the internal mammary artery 4 months after minimally invasive repair of pectus excavatum. J Pediatr Surg 43: 394-397.

8. Lin CW, Chen KC, Diau GY, Chu CC (2012) Late-onset vital complication after the Nuss procedure for pectus excavatum. Pediatr Surg Int 28: 71-73.

9. Fallon SC, Slater BJ, Nuchtern JG, Cass DL, Kim ES, et al. (2013) Complications related to the Nuss procedure: Minimizing risk with operative technique. J Pediatr Surg 48: 1044-1048. 